\title{
Nonlinear Second Order System of Neumann Boundary Value Problems at Resonance*
}

\author{
Chaitan P. Gupta ${ }^{\dagger}$ \\ Mathematics and Computer Science Division \\ Argonne National Laboratory \\ Argonne, IL 60439-4801
}

\begin{abstract}
Let $f:[0, \pi] \times \mathbf{R}^{N} \rightarrow \mathbf{R}^{N},(N \geq 1)$ satisfy Caratheodory conditions, $e(x) \in L^{1}\left([0, \pi] ; \mathbf{R}^{N}\right)$. This paper studies the system of nonlinear Neumann boundary value problems

$$
\begin{gathered}
x^{\prime \prime}(t)+f(t, x(t))=e(t), 0<t<\pi, \\
x^{\prime}(0)=x^{\prime}(\pi)=0 .
\end{gathered}
$$

This problem is at resonance since the associated linear boundary value problem

$$
\begin{gathered}
x^{\prime \prime}(t)=\lambda x(t), \quad 0<t<\pi, \\
x^{\prime}(0)=x^{\prime}(\pi)=0,
\end{gathered}
$$

has $\lambda=0$ as an eigenvalue. Asymptotic conditions on the nonlinearity $f(t, x(t))$ are offered to give existence of solutions for the nonlinear systems. The methods apply to the corresponding system of Lienard-type periodic boundary value problems.

Key words and phrases: Second-order system of Neumann boundary value problems, resonance at infinitely many eigenvalues, absence of $L^{\infty}$-resonance, asymptotic resonance conditions, Fredholm operator
\end{abstract}

AMS (MOS) Subject Classification: 34B15, 34B99

\section{Introduction}

Let $\mathbf{R}^{N}$ denote the $N$-dimensional Euclidean space. For $x=\left(x_{1}, x_{2}, \ldots, x_{N}\right) \in \mathbf{R}^{N}$, and $y=$ $\left(y_{1}, y_{2}, \ldots, y_{N}\right) \in \mathbf{R}^{N}$, let $|x|=\sqrt{x_{1}^{2}+x_{2}^{2}+\ldots+x_{N}^{2}}$, and $\langle x, y\rangle=x_{1} y_{1}+x_{2} y_{2}+\ldots+x_{N} y_{N}$ denote the Euclidean norm of $x$ and the inner product of $x$ and $y$ in $\mathbf{R}^{N}$, respectively. Let

*This work was supported by the Applied Mathematical Sciences subprogram of the Office of Energy Research, U. S. Department of Energy, under Contract W-31-109-Eng-38.

'Permanent address: Department of Mathematical Science, Northern Illinois University, DeKalb, IL 60115. 
$f=\left(f_{1}, f_{2}, \ldots, f_{N}\right):[0, \pi] \times \mathbf{R}^{N} \rightarrow \mathbf{R}^{N}$ be a function satisfying Caratheodory's conditions, and let $e:[0, \pi] \rightarrow \mathbf{R}^{N}$ be a Lebesgue integrable function.

This paper is devoted to the study of systems of Neumann boundary value problems

$$
\begin{array}{r}
-x^{\prime \prime}(t)+f(t, x(t))=e(t), \quad 0<t<\pi, \\
x^{\prime}(0)=x^{\prime}(\pi)=0,
\end{array}
$$

and

$$
\begin{array}{r}
x^{\prime \prime}(t)+f(t, x(t))=e(t), \quad 0<t<\pi, \\
x^{\prime}(0)=x^{\prime}(\pi)=0 .
\end{array}
$$

We obtain the existence of a solution for (1.1)-(1.2) when $\int_{0}^{\pi} e(t) d t=0$ and when, for each $i=$ $1,2, \ldots, N$, there exists a real number $r_{i} \geq 0$ such that

$$
\text { (i) } f_{i}(t, x) x_{i} \geq 0
$$

for a.e. $t \in[0, \pi]$ and all $x \in \mathrm{R}^{N}$ with $\left|x_{i}\right| \geq r_{i}$, and

$$
\text { (ii) }\left|f_{i}(t, x)\right| \leq \alpha_{i}(t)
$$

for a.e. $t \in[0, \pi]$ and all $x \in \mathrm{R}^{N}$ with $\left|x_{i}\right| \leq r_{i}$. We give asymptotic conditions on the behavior of $x_{i}^{-1} f_{i}(t, x), i=1,2, \ldots, N$, at the first two eigenvalues 0 and 1 of the linear problem

$$
\begin{gathered}
x^{\prime \prime}(t)+\lambda x(t)=0,0<t<\pi, \\
x^{\prime}(0)=x^{\prime}(\pi)=0,
\end{gathered}
$$

for the problem (1.3)-(1.4).

Our methods can be adapted and similar results obtained for Lienard's system of equations

$$
\begin{gathered}
\pm x^{\prime \prime}(t)+\left[\frac{d}{d t} \operatorname{grad} F(x(t))\right]+f(t, x(t))=e(t), \quad 0<t<\pi, \\
x(0)-x(2 \pi)=x^{\prime}(0)-x^{\prime}(2 \pi)=0,
\end{gathered}
$$

where $F: \mathbf{R}^{N} \rightarrow \mathbf{R}$ is in $C^{2}\left(\mathbf{R}^{N}, \mathbf{R}\right), f:[0,2 \pi] \times \mathbf{R}^{N} \rightarrow \mathbf{R}^{N}$ satisfies Caratheodory's conditions, and $e:[0,2 \pi] \rightarrow \mathbf{R}^{N}$ is Lebesgue integrable. The problem (1.9)-(1.10) was studied by Ianacci and Nkashama in [3], where they give sufficient non-resonance conditions for the existence of a solution. We provide in this paper sufficient resonance conditions for the existence of a solution for the problems (1.1)-(1.2) and (1.3)-(1.4) and accordingly for (1.9)-(1.10) in line with our remark above.

Our results and methods are inspired by the results of Gupta and Mawhin [2] for the problem (1.9)-(1.10) when $N=1$. We present in Section 2 notations and definitions that we need in this paper. In Section 3 we present some lemmas that are extensions to systems of corresponding lemmas in [3]. We present in Section 4 our theorems giving the existence of solutions for the problems (1.1)-(1.2) and (1.3)-(1.4). Our conditions for the existence of solutions for (1.3)-(1.4) allow resonance at infinitely many eigenvalues of the linear problem (1.7)-(1.8). Finally, in Section 5 we present a theorem for the problem (1.3)-(1.4) sharpening the condition for resonance at infinitely many eigenvalues of the linear problem (1.7)-(1.8) in the absence of $L^{\infty}$-resonance at the second eigenvalue $\lambda=1$ of (1.7)-(1.8). 


\section{Notations and Definitions}

Let $\mathbf{R}^{N}, N \geq 1$, denote the $N$-dimensional Euclidean space. For $x=\left(x_{1}, x_{2}, \ldots, x_{n}\right)$, let

$$
|x|=\left(x_{1}^{2}+x_{2}^{2}+\ldots+x_{N}\right)^{1 / 2}
$$

denote the Euclidean norm of $x$ in $\mathbf{R}^{N}$; and for $x=\left(x_{1}, x_{2}, \ldots, x_{N}\right)$ and $y=\left(y_{1}, y_{2}, \ldots, y_{N}\right)$ in $\mathbf{R}^{N}$, let

$$
<x, y>=\sum_{i=1}^{N} x_{i} y_{i}
$$

denote the inner product of $x$ and $y$ in $\mathbf{R}^{N}$.

We shall use the following spaces:

(i) the Lebesgue spaces $L^{p}\left([0, \pi], \mathbf{R}^{N}\right), 1 \leq p \leq \infty$, with the norms defined by

$$
\|f\|_{L_{N}^{p}}=\left[\sum_{i=1}^{N}\left(\frac{1}{\pi} \int_{0}^{\pi}\left|f_{i}\right|^{p} d t\right)^{2 / p}\right]^{1 / 2}, \text { for } 1 \leq p<\infty
$$

and

$$
\|f\|_{L_{N}^{\infty}}=\left(\sum_{i=1}^{N}\left|f_{i}\right|_{L^{\infty}}^{2}\right)^{1 / 2}, \text { for } p=\infty
$$

(ii) the space of $C\left([0, \pi], \mathrm{R}^{N}\right)$ of continuous functions with its usual norm, the norm induced by the Lebesgue space $L^{\infty}\left([0, \pi], \mathrm{R}^{N}\right)$;

(iii) the Sobolev space $H^{1}\left([0, \pi], \mathrm{R}^{N}\right)$ defined by

$$
H^{1}\left([0, \pi], \mathbf{R}^{N}\right)=\left\{x:[0, \pi] \rightarrow \mathbf{R}^{N} \mid x \text { is absolutely continuous and } x^{\prime} \in L^{2}\left([0, \pi], \mathbf{R}^{N}\right)\right\},
$$

with the inner product defined by

$$
(x, y)_{H_{N}^{1}}=<\frac{1}{\pi} \int_{0}^{\pi} x(t) d t, \frac{1}{\pi} \int_{0}^{\pi} y(t) d t>+\frac{1}{\pi} \int_{0}^{\pi}<x^{\prime}(t), y^{\prime}(t)>d t,
$$

and the corresponding norm $\|\cdot\|_{H_{N}^{1}}$ defined by

$$
\|x\|_{H_{N}^{1}}=\left(\frac{1}{\pi} \int_{0}^{\pi}\left|x^{\prime}(t)\right|^{2} d t+\left|\frac{1}{\pi} \int_{0}^{\pi} x(t) d t\right|^{2}\right)^{1 / 2}
$$

(iv) the Sobolev space $\tilde{H}^{1}\left([0, \pi], \mathbf{R}^{N}\right)$ defined by

$$
\tilde{H}^{1}\left([0, \pi], \mathbf{R}^{N}\right)=\left\{x \in H^{1}\left([0, \pi], \mathbf{R}^{N}\right) \mid \int_{0}^{\pi} x(t) d t=0\right\}
$$

with the norm induced by $H^{1}\left([0, \pi], \mathbf{R}^{N}\right)$; and

(v) the Sobolev space $W^{2,1}\left([0, \pi], \mathbf{R}^{N}\right)$ defined by

$$
W^{2,1}\left([0, \pi], \mathbf{R}^{N}\right)=\left\{x:[0, \pi] \rightarrow \mathbf{R}^{N} \mid x \text { and } x^{\prime} \text { absolutely continuous }\right\}
$$


with the norm defined by

$$
\|x\|_{W_{N}^{2,1}}=\sum_{j=0}^{2}\left\|x^{(j)}\right\|_{L_{N}^{1}}
$$

where $x^{(0)} \equiv x, x^{(1)}=x^{\prime}, x^{(2)}=x^{\prime \prime}$.

For the sake of simplicity in the notation of the space, we shall omit $\mathbf{R}^{N}$ when $N=1$.

We note that for $x \in H^{1}\left([0, \pi] ; \mathbf{R}^{N}\right), x=\left(x_{1}, x_{2}, \ldots, x_{N}\right)$ if and only if $x_{i} \in H^{1}[0, \pi]$, for $i=1,2, \ldots, N$. Also, every $x_{i} \in H^{1}[0, \pi]$ can be written in the form

$$
x_{i}(t)=\overline{x_{i}}+\tilde{x_{i}}(t)
$$

with $\tilde{x_{i}} \in \tilde{H}^{1}[0, \pi]$ and $\overline{x_{i}}=\frac{1}{\pi} \int_{0}^{\pi} x_{i}(t) d t$. Moreover,

$$
\left\|x_{i}\right\|_{H^{1}}=\left(\bar{x}_{i}^{2}+\frac{1}{\pi} \int_{0}^{\pi}\left(\tilde{x}_{i}^{\prime}(t)\right)^{2} d t\right)^{1 / 2}
$$

so that we have

$$
\|x\|_{H_{N}^{1}}=\left(\sum_{i=1}^{N}\left\|x_{i}\right\|_{H^{1}}^{2}\right)^{1 / 2}
$$

For $x=\left(x_{1}, x_{2}, \ldots, x_{N}\right) \in L^{1}\left[(0, \pi), \mathrm{R}^{N}\right]$, we write $\bar{x}=\left(\bar{x}_{1}, \ldots, \bar{x}_{N}\right)$, where $\bar{x}_{i}=\frac{1}{\pi} \int_{0}^{\pi} x_{i}(t) d t$, $i=1,2, \ldots, N$ and $\tilde{x}=x-\bar{x}$.

\section{Technical Lemmas}

Lemma 1 Let $\Gamma=\left(\Gamma_{1}, \Gamma_{2}, \ldots, \Gamma_{N}\right) \in L^{1}\left([0, \pi], \mathbf{R}^{N}\right)$ be such that for a.e. $t \in[0, \pi]$,

$$
\Gamma_{i}(t) \leq 1,
$$

for $i=1,2, \ldots, N$ with strict inequality holding on a subset of $[0, \pi]$ of positive measure. Then there exists a $\delta=\delta(\Gamma)>0$ such that for all $\tilde{x} \in \tilde{H}^{1}\left([0, \pi], \mathbf{R}^{N}\right)$ with $\tilde{x}^{\prime}(0)=\tilde{x}^{\prime}(\pi)=0$,

$$
\begin{aligned}
B_{\Gamma}(\tilde{x}) & =\frac{1}{\pi} \int_{0}^{\pi}\left[\left|\tilde{x}^{\prime}(t)\right|^{2}-\sum_{i=1}^{N} \Gamma_{i}(t) \tilde{x}_{i}^{2}(t)\right] d t \\
& \geq \delta\|\tilde{x}\|_{H_{N}^{1}}^{2} .
\end{aligned}
$$

Proof. Using (3.1), the method of expanding a scalar function $\tilde{x}_{i} \in \tilde{H}^{1}[0, \pi]$, with $\tilde{x}_{i}^{\prime}(0)=0$ and $\tilde{x}_{i}^{\prime}(\pi)=0$, into a cosine Fourier series, and Parseval's identities for $\tilde{x}_{i}$ and $\tilde{x}_{i}^{\prime}$, we see that

$$
\begin{aligned}
B_{\Gamma}(\tilde{x}) & =\frac{1}{\pi} \int_{0}^{\pi}\left[\left|\tilde{x}^{\prime}(t)\right|^{2}-\sum_{i=1}^{N} \Gamma_{i}(t) \tilde{x}_{i}^{2}(t)\right] d t \\
& =\sum_{i=1}^{N} \frac{1}{\pi} \int_{0}^{\pi}\left[\left(\tilde{x}_{i}^{\prime}(t)\right)^{2}-\Gamma_{i}(t) \tilde{x}_{i}^{2}(t)\right] d t \\
& \geq 0,
\end{aligned}
$$


for all $\tilde{x} \in \tilde{H}^{1}\left([0, \pi], \mathbf{R}^{N}\right)$ with $\tilde{x}^{\prime}(0)=\tilde{x}^{\prime}(\pi)=0$. Moreover,

$$
B_{\Gamma}(\tilde{x})=0
$$

if and only if

$$
\tilde{x}(t)=A \cos t
$$

for some $A=\left(A_{1}, A_{2}, \ldots, A_{N}\right) \in \mathrm{R}^{N}$. But we then get from (3.4) and (3.5) that

$$
0=B_{\Gamma}(\tilde{x})=\sum_{i=1}^{N} \frac{A_{i}^{2}}{\pi} \int_{0}^{\pi}\left(1-\Gamma_{i}(t)\right) \cos ^{2} t d t
$$

so that by our assumption (3.1) on $\Gamma_{i}$ we have $A_{i}=0$ for every $i=1,2, \ldots, N$, and hence $\tilde{x}=0$.

Let us next assume that the conclusion of the lemma is false. Then there exists a sequence $\left\{\tilde{x}_{n}\right\}, \tilde{x}_{n} \in \tilde{H}^{1}\left([0, \pi], \mathbf{R}^{N}\right)$, such that

$$
\begin{aligned}
B_{\Gamma}\left(\tilde{x}_{n}\right) & \rightarrow 0 \text { as } n \rightarrow \infty \\
\left\|\tilde{x}_{n}\right\|_{H_{N}^{1}} & =1, \text { for every } n=1,2, \ldots
\end{aligned}
$$

We may also assume, by going to a subsequence if necessary, that there exists an $\tilde{x} \in \tilde{H}^{1}\left([0, \pi], \mathbf{R}^{N}\right)$ such that

$$
\begin{aligned}
& \tilde{x}_{n} \rightarrow \tilde{x} \text { weakly in } H^{1}\left([0, \pi], \mathbf{R}^{N}\right), \\
& \tilde{x}_{n} \rightarrow \tilde{x} \text { in } C\left([0, \pi], \mathbf{R}^{N}\right) .
\end{aligned}
$$

Using Theorem 5.2 in [4], we have that $B_{\Gamma}(\tilde{x}) \geq 0$, even though $\tilde{x}^{\prime}(0)$ and $\tilde{x}^{\prime}(\pi)$ may not be zero. Also, from (3.7) and the weak lower semicontinuity of the norm in $H^{1}\left([0, \pi], \mathbf{R}^{N}\right)$, we have that

$$
\|\tilde{x}\|_{H_{N}^{1}} \leq \lim \inf _{n \rightarrow \infty}\left\|\tilde{x}_{n}\right\|_{H_{N}^{1}}=1
$$

and hence

$$
0 \leq B_{\Gamma}(\tilde{x}) \leq \lim \inf _{n \rightarrow \infty} B_{\Gamma}\left(\tilde{x}_{n}\right)=0 .
$$

Thus $\tilde{x}=0$, from the first part of the proof. We next see that

$$
\begin{aligned}
\frac{1}{\pi} \int_{0}^{\pi}\left|\tilde{x}_{n}^{\prime}\right|^{2} d t & =B_{\Gamma}\left(\tilde{x}_{n}\right)+\frac{1}{\pi} \sum_{i=1}^{N} \int_{0}^{\pi} \Gamma_{i}(t)\left|\tilde{x}_{n i}(t)\right|^{2} d t \\
& \rightarrow \frac{1}{\pi} \sum_{i=1}^{N} \int_{0}^{\pi} \Gamma_{i}(t)\left|\tilde{x}_{i}(t)\right|^{2} d t=\frac{1}{\pi} \int_{0}^{\pi}\left|\tilde{x}^{\prime}(t)\right|^{2} d t
\end{aligned}
$$

Thus, $\tilde{x}_{n} \rightarrow \tilde{x}$ in $H^{1}\left([0, \pi], \mathbf{R}^{N}\right)$ and $\|\tilde{x}\|_{H_{N}^{1}}=1$, which forms a contradiction. Hence the lemma.

Lemma 2 Let $\Gamma=\left(\Gamma_{1}, \Gamma_{2}, \ldots, \Gamma_{N}\right) \in L^{1}\left([0, \pi], \mathbf{R}^{N}\right)$ and $\Gamma_{\alpha}=\left(\Gamma_{\alpha_{1}}, \ldots, \Gamma_{\alpha_{N}}\right) \in L^{1}\left([0, \pi), \mathbf{R}^{N}\right)$. Let $\Gamma_{\beta}=\left(\Gamma_{\beta_{1}}, \ldots, \Gamma_{\beta_{N}}\right) \in L^{1}\left([0, \pi), \mathbf{R}^{N}\right)$ and $\Gamma_{\infty}=\left(\Gamma_{\infty_{1}}, \ldots, \Gamma_{\infty_{N}}\right) \in L^{\infty}\left([0, \pi], \mathbf{R}^{N}\right)$ be such that

$$
\text { (i) } \quad \Gamma=\Gamma_{\alpha}+\Gamma_{\beta}+\Gamma_{\infty}
$$

(ii) for a.e. $t \in[0, \pi]$ and every $i=1,2, \ldots, N, \Gamma_{\alpha_{i}}(t) \leq 1$, 
with strict inequality holding on a subset of $[0, \pi]$ of positive measure,

$$
\text { (iii) } \frac{\pi^{2}}{3}\left\|\Gamma_{\beta}\right\|_{L_{N}^{1}}+\left\|\Gamma_{\infty}\right\|_{L_{N}^{\infty}}<\delta\left(\Gamma_{\alpha}\right),
$$

where $\delta\left(\Gamma_{\alpha}\right)>0$ is given by Lemma 1 .

Then for every $\tilde{x} \in \tilde{H}^{1}\left([0, \pi], \mathbf{R}^{N}\right)$ with $\tilde{x}^{\prime}(0)=\tilde{x}^{\prime}(\pi)=0$,

$$
B_{\Gamma}(\tilde{x}) \geq\left[\delta\left(\Gamma_{\alpha}\right)-\frac{\pi^{2}}{3}\left\|\Gamma_{\beta}\right\|_{L_{N}^{1}}-\left\|\Gamma_{\infty}\right\|_{L_{N}^{\infty}}\right]\|\tilde{x}\|_{H_{N}^{1}}^{2}
$$

Proof. Using the fact that $H^{1}\left([0, \pi], \mathbf{R}^{N}\right) \subset C\left([0, \pi], \mathbf{R}^{N}\right)$ and the inequalities (see [8])

$$
\|\tilde{x}\|_{L_{N}^{2}} \leq\left\|\tilde{x}^{\prime}\right\|_{L_{N}^{2}} \leq\|\tilde{x}\|_{H_{N}^{1}},\|\tilde{x}\|_{L_{N}^{\infty}} \leq \frac{\pi}{\sqrt{3}}\left\|\tilde{x}^{\prime}\right\|_{L_{N}^{2}} \leq \frac{\pi}{\sqrt{3}}\|\tilde{x}\|_{H_{N}^{1}}
$$

for all $\tilde{x} \in \tilde{H}^{1}\left([0, \pi] ; \mathbf{R}^{N}\right)$, as well as Lemma 1 , we see that

$$
\begin{aligned}
B_{\Gamma}(\tilde{x})= & \frac{1}{\pi} \int_{0}^{\pi}\left[\left|\tilde{x}^{\prime}(t)\right|^{2}-\sum_{i=1}^{N} \Gamma_{i}(t) \tilde{x}_{i}^{2}(t)\right] d t \\
= & \frac{1}{\pi} \int_{0}^{\pi}\left[\left|\tilde{x}^{\prime}(t)\right|^{2}-\sum_{i=1}^{N} \Gamma_{\alpha_{i}}(t) \tilde{x}^{2}(t)\right] d t \\
& -\frac{1}{\pi} \sum_{i=1}^{N} \int_{0}^{\pi}\left[\Gamma_{\beta_{i}}(t)+\Gamma_{\infty_{i}}(t)\right] \tilde{x}_{i}^{2}(t) d t \\
\geq & \delta\left(\Gamma_{\alpha}\right)\|\tilde{x}\|_{H_{N}^{1}}^{2}-\left\|\Gamma_{\beta}\right\|_{L_{N}^{1}}\|\tilde{x}\|_{L_{N}^{\infty}}^{2}-\left\|\Gamma_{\infty}\right\|_{L_{N}^{\infty}}\|\tilde{x}\|_{L_{N}^{2}}^{2} \\
\geq & \left(\delta\left(\Gamma_{\alpha}\right)-\frac{\pi^{2}}{3}\left\|\Gamma_{\beta}\right\|_{L_{N}^{1}}-\left\|\Gamma_{\infty}\right\|_{L_{N}^{\infty}}\right)\|\tilde{x}\|_{H_{N}^{1}}^{2} .
\end{aligned}
$$

Definition 1 For $x=\left(x_{1}, x_{2}, \ldots, x_{N}\right)$ and $y=\left(y_{1}, y_{2}, \ldots, y_{N}\right)$ in $\mathbf{R}^{N}$, we say $x \leq y$ if $x_{i} \leq y_{i}$ for every $i=1,2, \ldots, N$.

Lemma 3 Let $\gamma=\left(\gamma_{1}, \gamma_{2}, \ldots, \gamma_{N}\right) \in L^{1}\left([0, \pi], \mathbf{R}^{N}\right)$ and $\Gamma=\Gamma_{\alpha}+\Gamma_{\beta}+\Gamma_{\infty} \in L^{1}\left([0, \pi], \mathbf{R}^{N}\right)$ be as in Lemma 2 , and let $\delta\left(\Gamma_{\alpha}\right)$ be given by Lemma 1. Then for all measurable functions $p:[0, \pi] \rightarrow \mathbf{R}^{N}$ such that $\bar{\gamma} \leq \bar{p}, p(t) \leq \Gamma(t)$ for a.e. $t \in[0, \pi]$ and all $x \in W^{2,1}\left[(0, \pi), \mathrm{R}^{N}\right]$ with $x^{\prime}(0)=x^{\prime}(\pi)=0$,

$$
\begin{gathered}
\frac{1}{\pi} \int_{0}^{\pi}<\bar{x}-\tilde{x}(t), \tilde{x}^{\prime \prime}(t)+p^{T}(t) I \tilde{x}(t)>d t \\
\geq \eta|\bar{x}|^{2}+\left[\delta\left(\Gamma_{\alpha}\right)-\frac{\pi^{2}}{3}\left\|\Gamma_{\beta}\right\|_{L_{N}^{1}}-\left\|\Gamma_{\infty}\right\|_{L_{N}^{\infty}}\right]\|\tilde{x}\|_{H_{N}^{1}}^{2} .
\end{gathered}
$$

Here $\eta=\min \left\{\bar{\gamma}_{i} \mid 1 \leq i \leq N\right\}, I$ denotes the $N \times N$ identity matrix, $p^{T}(t)$ denotes the transpose of the column vector col. $\left\{p_{1}(t), p_{2}(t), \ldots, p_{N}(t)\right\}$, and all vectors are understood as column vectors for the purpose of matrix arithmetic. 
Proof. For $x=\left(x_{1}, x_{2}, \ldots x_{N}\right) \in W^{2,1}\left([0, \pi], \mathbf{R}^{N}\right)$ with $x^{\prime}(0)=x^{\prime}(\pi)=0$, we have (on integrating by parts and from Lemma 2 ) that

$$
\begin{aligned}
\frac{1}{\pi} \int_{0}^{\pi} & <\bar{x}-\tilde{x}(t), \tilde{x}^{\prime \prime}(t)+p^{T}(t) I \tilde{x}(t)>d t \\
& =\frac{1}{\pi} \int_{0}^{\pi}\left|\tilde{x}^{\prime}(t)\right|^{2} d t+\sum_{i=1}^{N} \frac{1}{\pi} \int_{0}^{\pi} p_{i}(t)\left(\bar{x}_{i}^{2}-\tilde{x}_{i}^{2}(t)\right) d t \\
& =\frac{1}{\pi} \int_{0}^{\pi}\left[\left|\tilde{x}^{\prime}(t)\right|^{2}-\sum_{i=1}^{N} p_{i}(t) \tilde{x}_{i}^{2}(t)\right] d t+\sum_{i=1}^{N} \bar{p}_{i} \bar{x}_{i}^{2} \\
& \geq \sum_{i=1}^{N} \bar{\gamma}_{i} \bar{x}_{i}^{2}+\left[\delta\left(\Gamma_{\alpha}\right)-\frac{\pi^{2}}{3}\left\|\Gamma_{\beta}\right\|_{L_{N}^{1}}-\left\|\Gamma_{\infty}\right\|_{L_{N}^{\infty}}\right]\|\tilde{x}\|_{H_{N}^{1}}^{2} \\
& \geq \eta|\bar{x}|^{2}+\left[\delta\left(\Gamma_{\alpha}\right)-\frac{\pi^{2}}{3}\left\|\Gamma_{\beta}\right\|_{L_{N}^{1}}-\left\|\Gamma_{\infty}\right\|_{\left.L_{N}^{\infty}\right]}\right]\|\tilde{x}\|_{H_{N}^{1}}^{2} .
\end{aligned}
$$

Hence the lemma follows.

\section{Asymptotic Resonance Conditions for the Existence of Solu- tions}

Let $f:[0, \pi] \times \mathrm{R}^{N} \rightarrow \mathrm{R}^{N}$ be a function satisfying Caratheodory's conditions, namely,

(i) for each $x \in \mathbf{R}^{N}$, the function $t \in[0, \pi] \rightarrow f(t, x) \in \mathbf{R}^{N}$ is measurable on $[0, \pi]$;

(ii) for a.e. $t \in[0, \pi]$, the function $x \in \mathrm{R}^{N} \rightarrow f(t, x) \in \mathrm{R}^{N}$ is continuous; and

(iii) for each $r>0$, there exists a function $\alpha_{r}(t) \in L^{1}[0, \pi]$ such that $|f(t, x)| \leq \alpha_{r}(t)$ for a.e. $t \in[0, \pi]$ and all $x \in \mathbf{R}^{N}$ with $|x| \leq r$.

Let $x=C\left([0, \pi], \mathrm{R}^{N}\right)$ and $Y=L^{1}\left([0, \pi], \mathrm{R}^{N}\right)$. Also, let $Y_{1} \subset Y$ denote the subspace of $Y$ defined by

$$
Y_{1}=\left\{x \in L^{1}\left([0, \pi], \mathbf{R}^{N}\right) \mid x_{i}(t) \text { is constant for a.e. } t \in[0, \pi], i=1,2, \ldots, N\right\} ;
$$

and let $Y_{2}$ be the closed subspace of $Y$ such that $Y=Y_{1} \oplus Y_{2}$. We define the canonical projections $P: Y \rightarrow Y_{1}$ and $Q: Y \rightarrow Y_{2}$ by setting, for $x \in Y$,

$$
\begin{aligned}
& P x(t)=x(t)-\frac{1}{\pi} \int_{0}^{\pi} x(t) d t=\tilde{x}(t), \\
& Q x(t)=\frac{1}{\pi} \int_{0}^{\pi} x(t) d t=\bar{x}
\end{aligned}
$$

for $t \in[0, \pi]$.

We next define a linear operator $L: D(L) \subset X \rightarrow Y$ by setting

$$
D(L)=\left\{x \in W^{2,1}\left([0, \pi], \mathbf{R}^{N}\right) \mid x^{\prime}(0)=x^{\prime}(\pi)=0\right\} ;
$$

and for $x \in D(L)$,

$$
L x=-x^{\prime \prime}
$$


Now, for $x \in D(L)$, we see, on integrating by parts, that

$$
\begin{aligned}
(L x, x) & =\frac{1}{\pi} \int_{0}^{\pi}\left\langle-x^{\prime \prime}(t), x(t)>d t\right. \\
& =\frac{1}{\pi} \int_{0}^{\pi}\left|x^{\prime}(t)\right|^{2} d t=\|\tilde{x}\|_{H_{N}^{1}}^{2} \geq 0 .
\end{aligned}
$$

Lemma 4 For every given $y \in L^{1}\left([0, \pi], \mathbf{R}^{N}\right)$ with $\bar{y}=0$, there exists a unique $x \in C\left([0, \pi], \mathbf{R}^{N}\right)$ with $\bar{x}=0$ such that

$$
\begin{gathered}
-x^{\prime \prime}(t)=y(t), \quad 0<t<\pi, \\
x^{\prime}(0)=x^{\prime}(\pi)=0 .
\end{gathered}
$$

Proof. It is easy to see that

$$
x(t)=-\int_{0}^{t}(t-\tau) y(\tau) d \tau+\frac{1}{2 \pi} \int_{0}^{\pi}(\pi-\tau)^{2} y(\tau) d \tau,
$$

for $t \in[0, \pi]$, is the unique solution for (4.7)-(4.8) with $\bar{x}=0$. Hence the lemma follows.

It follows from Lemma 4 that there is a bounded linear operator $K: Y_{1} \rightarrow X$ such that for $y \in Y$,

$$
K P y \in D(L), L K P y=P y, \quad(K P y, P y) \geq 0 .
$$

Now let $N: X \rightarrow Y$ be a nonlinear operator defined by

$$
(N x)(t)=f(t, x(t)), t \in[0, \pi],
$$

where $f:[0, \pi] \times \mathbf{R}^{N} \rightarrow \mathbf{R}^{N}$ is a given function satisfying Caratheodory's conditions. It follows easily from the Arzela-Ascoli theorem that the operator $K P N: X \rightarrow X$ is a compact operator (i.e., it maps bounded subsets in $X$ into relatively compact subsets of $X$ ) and $Q N: X \rightarrow X$ is a bounded operator (i.e., $Q N$ maps bounded subsets in $X$ into bounded subsets in $X$ ).

Theorem 1 Let $f=\left(f_{1}, f_{2}, \ldots, f_{N}\right):[0, \pi] \times \mathbf{R}^{N} \rightarrow \mathbf{R}$ be a function satisfying Caratheodory's conditions. Suppose that for each $i=1,2, \ldots, N$ there exist real numbers $r_{i}, R_{i}, a_{i}$ and $A_{i}$ with $r_{i}<0<R_{i}$ and $a_{i} \leq A_{i}$ such that

(i) for a.e. $t \in[0, \pi]$ and all $x \in \mathrm{R}^{N}$ with $x_{i} \geq R_{i}$,

$$
f_{i}(t, x) \geq A_{i}
$$

(ii) for a.e. $t \in[0, \pi]$ and all $x \in \mathbf{R}^{N}$ with $x_{i} \leq r_{i}$,

$$
f_{i}(t, x) \leq a_{i} .
$$

Suppose further that for every real number $r \geq 0$ and each $i=1,2, \ldots, N$ there exist functions $\alpha_{r i}(t) \in L^{1}[0, \pi]$ such that

$$
\left|f_{i}(t, x)\right| \leq \alpha_{r i}(t)
$$


for a.e. $t \in[0, \pi]$ and all $x \in \mathbf{R}^{N}$ with $\left|x_{i}\right| \leq r$. Then for every $e \in L^{1}\left([0, \pi], \mathbf{R}^{N}\right)$ with $a_{i} \leq \bar{e}_{i} \leq A_{i}$ for each $i=1,2, \ldots, N$ the boundary value problem

$$
\begin{gathered}
-x^{\prime \prime}(t)+f(t, x(t))=e(t), \quad 0<t<\pi, \\
x^{\prime}(0)=x^{\prime}(\pi)=0,
\end{gathered}
$$

has at least one solution.

Proof. Define $F=\left(F_{1}, F_{2}, \ldots, F_{N}\right):[0, \pi] \times \mathrm{R}^{N} \rightarrow \mathrm{R}^{N}$ by

$$
F_{i}(t)=f_{i}(t)-\frac{A_{i}+a_{i}}{2}
$$

for $(t, x) \in[0, \pi] \times \mathbf{R}^{N}$ and $i=1,2, \ldots, N$. Also define $E:[0, \pi] \rightarrow \mathbf{R}^{N}, E=\left(E_{1}, E_{2}, \ldots, E_{N}\right)$, by

$$
E_{i}(t)=e_{i}(t)-\frac{A_{i}+a_{i}}{2}
$$

for $t \in[0, \pi]$ and $i=1,2, \ldots, N$. Clearly $F$ satisfies Caratheodory's conditions, and for $i=1,2, \ldots, N$ and for a.e. $t \in[0, \pi]$,

$$
F_{i}(t, x) \geq \frac{A_{i}-a_{i}}{2} \geq 0
$$

for all $x \in \mathbf{R}^{N}$ with $x_{i} \geq R_{i}$, while

$$
F_{i}(t, x) \leq \frac{A_{i}-A_{i}}{2} \leq 0
$$

for all $x \in \mathrm{R}^{N}$ with $x_{i} \leq r_{i}$. Further, for every real number $r \geq 0$ and each $i=1,2, \ldots, N$ there exist functions $\beta_{r i} \in L^{1}[0, \pi]$ such that

$$
\left|F_{i}(t, x)\right| \leq \beta_{r i}(t)
$$

for a.e. $t \in[0, \pi]$ and all $x \in \mathbf{R}^{N}$ with $\left|x_{i}\right| \leq r$. Indeed, in view of (4.14), we have $\beta_{r i}(t)=$ $\alpha_{r i}(t)+\frac{1}{2}\left|A_{i}+a_{i}\right|$ for $t \in[0, \pi]$. We also have for $i=1,2, \ldots, N$,

$$
\frac{1}{2}\left(a_{i}-A_{i}\right) \leq \bar{E}_{i} \leq \frac{1}{2}\left(A_{i}-a_{i}\right)
$$

Clearly, (4.15) is equivalent to

$$
-x^{\prime \prime}(t)+F(t, x(t))=E(t), 0<t<\pi .
$$

Let us next define the nonlinear operator $N: X \rightarrow Y$ by

$$
(N x)(t)=F(t, x(t)), \quad t \in[0, \pi]
$$

while $x(t) \in X$. It is easy to see, in view of (4.19), (4.20), and (4.21), that for every $k \geq 0$ there exists a constant $C(k) \geq 0$ such that

$$
(N x, x) \geq k\|N x\|_{Y}-C(k)
$$


for $x \in X$. Now, if $L: D(L) \subset X \rightarrow Y$ is the linear operator defined by (4.4) and (4.5) and $K: Y_{1} \rightarrow X$ is the linear operator as in (4.10), then the boundary value problem of (4.23) with (4.16) is equivalent to the operator equation

$$
L x+N x=E, \quad x \in X
$$

which in turn is equivalent to the system of equations

$$
\begin{aligned}
P x+K P N x & =K P E \\
Q N x & =Q E,
\end{aligned}
$$

where $P$ and $Q$ are as defined by (4.2) and (4.3). Now, (4.26) is clearly equivalent to the single equation

$$
P x+Q N x+K P N x=K P E+Q E,
$$

which has the form of a compact perturbation of the Fredholm operator $P$ of index zero. We can, therefore, apply the version given in [6: Theorem 1, Corollary 1] or [5: Theorem IV.4] or [7] of the Leray-Schauder Continuation theorem which ensures the existence of a solution for (4.27) if the set of all possible solutions of the family of equations

$$
P x+(1-\lambda) Q x+\lambda Q N x+\lambda K P N=\lambda K P E+\lambda Q E,
$$

$\lambda \in(0,1)$, is a priori bounded in $X$, independently of $\lambda$. Notice that $(4.28)$ is equivalent to the system of equations

$$
\begin{aligned}
P x+\lambda K P N x & =\lambda K P E \\
(1-\lambda) Q x+\lambda Q N x & =\lambda Q E .
\end{aligned}
$$

If $x_{\lambda} \in X$ is a solution for (4.29) for some $\lambda \in(0,1)$, then $x_{\lambda} \in D(L)$ and

$$
\begin{aligned}
L x_{\lambda}+\lambda P N x_{\lambda} & =\lambda P E \\
(1-\lambda) Q x_{\lambda}+\lambda Q N x_{\lambda} & =\lambda Q E .
\end{aligned}
$$

Now we get from (4.30) that

$$
\begin{gathered}
\left(L x_{\lambda}, P x_{\lambda}\right)+\lambda\left(P N x_{\lambda}, P x_{\lambda}\right)=\lambda\left(P E, P x_{\lambda}\right), \\
(1-\lambda)\left(Q x_{\lambda}, Q x_{\lambda}\right)+\lambda\left(Q N x_{\lambda}, Q x_{\lambda}\right)=\lambda\left(Q E, Q x_{\lambda}\right) .
\end{gathered}
$$

Since $\left(L x_{\lambda}, P x_{\lambda}\right)=\left(L x_{\lambda}, x_{\lambda}\right)$, and given (4.6) and (4.24), we obtain that

$$
\left\|P x_{\lambda}\right\|_{H_{N}^{1}}^{2}+k\left\|N x_{\lambda}\right\|_{Y}-C(k) \leq\|P E\|_{Y} \cdot\left\|P x_{\lambda}\right\|_{X}+|Q E| \cdot\left|Q x_{\lambda}\right| .
$$

Now, the second equation in (4.30) gives for each $i=1,2, \ldots, N$ that

$$
(1-\lambda) \frac{1}{\pi} \int_{0}^{\pi} x_{\lambda i}(t) d t+\lambda \frac{1}{\pi} \int_{0}^{\pi} F_{i}\left(t, x_{\lambda}(t)\right) d t=\lambda \frac{1}{\pi} \int_{0}^{\pi} E_{i}(t) d t .
$$

If $x_{\lambda i}(t) \geq R_{i}$ for every $t \in[0, \pi]$, we get from (4.32), in view of (4.19) and (4.22), that

$$
(1-\lambda) R_{i}+\frac{\lambda}{2}\left(A_{i}-a_{i}\right) \leq \frac{\lambda}{2}\left(A_{i}-a_{i}\right)
$$


Thus, $(1-\lambda) R_{i} \leq 0$, and we have a contradiction. Similarly, $x_{\lambda i}(t) \leq r_{i}$ for every $t \in[0, \pi]$ leads to a contradiction. So for every $i=1,2, \ldots, N$ there exists a $\tau_{i} \in[0, \pi]$ such that $r_{i} \leq x_{\lambda i}\left(\tau_{i}\right) \leq R_{i}$. It follows that there exist constants $C_{1} \geq 0$ and $C_{2} \geq 0$, independent of $\lambda \in(0,1)$ such that

$$
\left\|x_{\lambda}\right\|_{X} \leq C_{1}+C_{2}\left\|P x_{\lambda}\right\|_{H_{N}^{1}}
$$

Finally, using the facts that $\left\|P x_{\lambda}\right\|_{X} \leq 2\left\|x_{\lambda}\right\|_{X}$ and $\left|Q x_{\lambda}\right| \leq\left\|x_{\lambda}\right\|_{X}$, we have that

$$
\left\|P x_{\lambda}\right\|_{H_{N}^{1}}^{2}+k\left\|N x_{\lambda}\right\|_{Y}-C(k) \leq C_{3}\left(C_{1}+C_{2}\left\|P x_{\lambda}\right\|_{H_{N}^{1}}\right)
$$

where $C_{3}=2\|P E\|_{Y}+|Q E|$. Hence, there exists a constant $C>0$, independent of $\lambda \in(0,1)$, such that

which implies, from (4.33), that

$$
\left\|P x_{\lambda}\right\|_{H_{N}^{1}} \leq C
$$

$$
\left\|x_{\lambda}\right\|_{X} \leq C_{1}+C_{2} C .
$$

We have thus shown that the set of solutions of $(4.28)$ is bounded in $X$ independently of $\lambda \in(0,1)$. Hence the theorem follows.

Theorem 2 Let $\Gamma=\left(\Gamma_{1}, \Gamma_{2}, \ldots, \Gamma_{N}\right) \in L^{1}\left([0, \pi], R^{N}\right)$ be as in Lemma 2. Let $f=\left(f_{1}, f_{2}, \ldots, f_{N}\right)$ : $[0, \pi] \times \mathbf{R}^{N} \rightarrow \mathbf{R}^{N}$ be as in Theorem 1. Assume, further, for each $i=1,2, \ldots, N$

$$
\lim \sup _{\left|x_{i}\right| \rightarrow \infty} \frac{f_{i}(t, x)}{x_{i}} \leq \Gamma_{i}(t),
$$

uniformly a.e. in $t \in[0, \pi]$. Then, for every $e \in L^{1}\left([0, \pi], \mathbf{R}^{N}\right)$ with $a_{i} \leq \bar{e}_{i} \leq A_{i}$ for each $i=1,2, \ldots, N$ the boundary value problem

$$
\begin{gathered}
x^{\prime \prime}(t)+f(t, x(t))=e(t), \quad 0<t<\pi, \\
x^{\prime}(0)=x^{\prime}(\pi)=0,
\end{gathered}
$$

has at least one solution.

Proof. Define $F=\left(F_{1}, F_{2}, \ldots, F_{n}\right):[0, \pi] \times \mathbf{R}^{N} \rightarrow \mathbf{R}^{N}$ and $E:[0, \pi] \rightarrow \mathbf{R}^{N}, E=\left(E_{1}, E_{2}, \ldots, E_{N}\right)$, as in the proof of Theorem 1, so that (4.19), (4.20), (4.21), and (4.22) hold. We have from (4.34) for each $i=1,2, \ldots, N$ that

$$
\lim \sup _{\left|x_{i}\right| \rightarrow \infty} \frac{F_{i}(t, x)}{x_{i}} \leq \Gamma_{i}(t),
$$

uniformly a.e. in $t \in[0, \pi]$. We also see for each $i=1,2, \ldots N$ for all $x \in \mathbf{R}^{N}$ with $\left|x_{i}\right| \geq$ $\max \left(R_{i},-r_{i}\right)$ that $F_{i}(t, x) x_{i} \geq 0$, so that $\Gamma_{i}(t) \geq 0$ a.e. in $[0, \pi]$. Moreover, the equation (4.35) is equivalent to

$$
x^{\prime \prime}(t)+F(t, x(t))=E(t), \quad 0<t<\pi .
$$

Now let $\eta=\frac{1}{2 N}\left[\delta\left(\Gamma_{\alpha}\right)-\frac{\pi^{2}}{3}\left\|\Gamma_{\beta}\right\|_{L_{N}^{1}}-\left\|\Gamma_{\infty}\right\|_{L_{N}^{\infty}}\right]>0$. Then for each $i$ there exists a $\rho_{i}>0$ such that for a.e. $t \in[0, \pi]$ and all $x \in \mathbf{R}^{N}$ with $\left|x_{i}\right| \geq \rho_{i}$,

$$
0 \leq \frac{F_{i}(t, x)}{x_{i}} \leq \Gamma_{i}(t)+\eta .
$$


Next, set $\rho=\max \left\{\rho_{i} \mid 1 \leq i \leq N\right\}$ and define $\tilde{\gamma}:[0, \pi] \times \mathbf{R}^{N}-\mathbf{R}^{N}$ by setting, for $(t, x) \in[0, \pi] \times \mathbf{R}^{N}$ and each $i=1,2, \ldots N$,

$$
\begin{aligned}
\tilde{\gamma}_{i}(t, x)= & \frac{F_{i}(t, x)}{x_{i}} \text { if }\left|x_{i}\right| \geq \rho, \\
\tilde{\gamma}_{i}(t, x)= & \frac{F_{i}\left(t, x_{1}, \ldots, x_{i-1}, \rho, x_{i+1}, \ldots, x_{N}\right)}{\rho}\left(\frac{x_{i}}{\rho}\right) \\
& +\left(1-\frac{x_{i}}{\rho}\right) \Gamma_{i}(t) \text { for } 0 \leq x_{i}<\rho, \\
\tilde{\gamma}_{i}(t, x)= & \frac{F_{i}\left(t, x_{1}, \ldots, x_{i-1},-\rho, x_{i+1}, \ldots, x_{N}\right)}{\rho}\left(\frac{x_{i}}{\rho}\right) \\
& +\left(1+\frac{x_{i}}{\rho}\right) \Gamma_{i}(t) \text { for }-\rho \leq x_{i}<0 .
\end{aligned}
$$

Then $\tilde{\gamma}$ satisfies Caratheodory's conditions and

$$
0 \leq \tilde{\gamma}_{i}(t, x) \leq \Gamma_{i}(t)+\eta
$$

for a.e. $t \in[0, \pi]$, all $x \in \mathbf{R}^{N}$, and $i=1,2, \ldots, N$. If we next set $h=\left(h_{1}, h_{2}, \ldots, h_{N}\right)$ with

$$
h_{i}(t, x)=F_{i}(t, x)-\tilde{\gamma}_{i}(t, x) x_{i},
$$

for $t \in[0, \pi], x \in \mathrm{R}^{N}$, and $i=1,2, \ldots, N$, then we see from (4.21) and the definition of $\tilde{\gamma}_{i}$ that there exist functions $m_{i}(t) \in L^{1}[0, \pi]$ such that

$$
\left|h_{i}(t, x)\right| \leq m_{i}(t)
$$

for a.e. $t \in[0, \pi]$ and all $x \in \mathbf{R}^{N}$. Defining $g:[0, \pi] \times \mathbf{R}^{N} \rightarrow \mathbf{R}^{N}, g=\left(g_{1}, g_{2}, \ldots, g_{N}\right)$ by setting

$$
g_{i}(t, x)=\tilde{\gamma}_{i}(t, x) x_{i}
$$

for $(t, x) \in[0, \pi] \times \mathrm{R}^{N}, i=1,2, \ldots, N$, we see that the equation (4.38) is equivalent to

$$
x^{\prime \prime}(t)+g(t, x(t))+h(t, x(t))=E(t) .
$$

We can next apply Theorem IV.4 of [5] to the boundary value problem posed by(4.42) and (4.36). It suffices to show that the set of solutions of the family of equations

$$
\begin{gathered}
x^{\prime \prime}(t)+(1-\lambda) \tilde{\Gamma}^{T}(t) I x(t)+\lambda g(t, x(t))+\lambda h(t, x(t))=\lambda E(t), \\
x^{\prime}(0)=x^{\prime}(\pi)=0,
\end{gathered}
$$

$\lambda \in(0,1)$, is a priori bounded in $X=C\left([0, \pi], \mathbf{R}^{N}\right)$ independently of $\lambda$, where $\tilde{\Gamma}(t)=\left(\tilde{\Gamma}_{1}(t), \ldots, \tilde{\Gamma}_{N}(t)\right)$ with $\tilde{\Gamma}_{i}(t)=\Gamma_{i}(t)+\eta, i=1,2, \ldots, N$.

If, now, $x(t)$ is a possible solution of (4.43) for some $\lambda \in(0,1)$, we see on integrating the equation obtained by taking the inner product of the equation in (4.43) with $\frac{1}{\pi}(\bar{x}-\tilde{x}(t))$ and using Lemma 3 with $\Gamma_{\infty i}$ replaced by $\Gamma_{\infty i}+\eta$, for $i=1,2, \ldots, N$ and $\gamma=\left(\gamma_{1}, \ldots, \gamma_{N}\right) \equiv 0$ that

$$
0=\frac{1}{\pi} \int_{0}^{\pi}\left\langle x^{\prime \prime}(t), \bar{x}-\tilde{x}(t)>d t\right.
$$




$$
\begin{aligned}
& +\frac{1}{\pi} \int_{0}^{\pi}\left\{\left\langle\bar{x}-\tilde{x}(t),(1-\lambda) \tilde{\Gamma}^{T}(t) I x(t)+\lambda g(t, x(t))+\lambda h(t, x(t))-\lambda E(t)>\right\} d t\right. \\
\geq & {\left[\delta\left(\Gamma_{\alpha}\right)-\frac{\pi^{2}}{3}\left\|\Gamma_{\beta}\right\|_{L_{N}^{1}}-\left\|\Gamma_{\infty}\right\| \|_{N}^{\infty}-N \eta\right]\|\tilde{x}\|_{H_{N}^{1}}^{2} } \\
& -\left[\sum_{i=1}^{N}\left\|m_{i}\right\|_{L^{1}}+\|E\|_{L_{N}^{1}}\right]\left(|\bar{x}|+\|\tilde{x}\|_{L_{N}^{\infty}}\right) \\
\geq & \eta N\|\tilde{x}\|_{H_{N}^{1}}^{2}-C\left(|\bar{x}|+\|\tilde{x}\|_{H_{N}^{1}}\right),
\end{aligned}
$$

where $C>0$ is a constant independent of $\lambda \in(0,1)$. Hence,

$$
\|\tilde{x}\|_{H_{N}^{1}}^{2} \leq\left(\frac{C}{\eta N}\right)\left(|\bar{x}|+\|\tilde{x}\|_{H_{N}^{1}}\right) .
$$

Next, integrating each of the component equations in $(4.43)$ over $[0, \pi]$, we see that

$$
\begin{gathered}
(1-\lambda) \frac{1}{\pi} \int_{0}^{\pi}\left(\Gamma_{i}(t)+\eta\right) x_{i}(t) d t+\lambda \frac{1}{\pi} \int_{0}^{\pi} F_{i}(t, x(t)) d t \\
=\lambda \frac{1}{\pi} \int_{0}^{\pi} E_{i}(t) d t
\end{gathered}
$$

$i=1,2, \ldots N$. As in the proof of Theorem 1 , we see that there exist constants $C_{1} \geq 0$ and $C_{2} \geq 0$, such that

$$
|\bar{x}| \leq\|x\|_{X} \leq C_{1}+C_{2}\|\tilde{x}\|_{H_{N}^{1}} .
$$

It follows from (4.44) and (4.45) that there exists a constant $C_{3}$ independent of $\lambda \in(0,1)$ such that

$$
\|\tilde{x}\|_{H_{N}^{1}} \leq C_{3},
$$

and hence

$$
\|x\|_{X} \leq C_{1}+C_{2} C_{3} .
$$

Thus we have shown that the set of solutions of (4.43) is bounded in $X$ independently of $\lambda$. Hence the theorem holds.

Remark 1. We say that the boundary value problem (4.35)-(4.36) has "no $L^{\infty}$-resonance" at the second eigenvalue $\lambda=1$ of the linear eigenvalue problem (1.7)-(1.8) if $\Gamma_{\alpha}=\Gamma_{\infty}=0$ in Theorem 2 . In the case of no $L^{\infty}$-resonance, Theorem 2 implies the existence of a solution for the boundary value problem (4.35)-(4.36) if $\left\|\Gamma_{\beta}\right\|_{L_{N}^{1}}<\frac{3}{\pi^{2}}$. We give a sharpening of this result in Section 5.

\section{Resonance Condition When No $L^{\infty}$-Resonance Exists}

We need the following lemma for a sharper resonance condition that gives the existence of a solution for the boundary value problem (4.35)-(4.36) when there is no $L^{\infty}$-resonance.

Lemma 5 Let $\in L^{1}\left([0, \pi], \mathbf{R}^{N}\right)$ and $\Gamma=\left(\Gamma_{1}, \Gamma_{2}, \ldots, \Gamma_{N}\right) \in L^{1}\left([0, \pi], \mathbf{R}^{N}\right)$ with $\bar{\Gamma}_{i}=\frac{1}{\pi} \int_{0}^{\pi} \Gamma_{i}(t) d t \geq$ 0 for every $i=1,2, \ldots, N$. Then every possible solution $x(t)$ of the linear boundary value problem

$$
x^{\prime \prime}(t)+p(t)^{T} I x(t)=e(t), 0<t<\pi
$$




$$
x^{\prime}(0)=x^{\prime}(\pi)=0,
$$

with $p=\left(p_{1}, p_{2}, \ldots, p_{N}\right) \in L^{1}\left([0, \pi], \mathrm{R}^{N}\right)$ such that

$$
\bar{p}_{i} \leq \bar{\Gamma}_{i}, \quad 0 \leq p_{i}(t)
$$

for a.e. $t \in[0, \pi], i=1,2, \ldots, N$, satisfies the inequality

$$
\left(1-\frac{\pi^{2}}{4}|\bar{\Gamma}|\right)\left\|x^{\prime \prime}\right\|_{L_{N}^{1}}^{2} \leq 2\|e\|_{L_{N}^{1}}\left\|x^{\prime \prime}\right\|_{L_{N}^{1}}+|\bar{\Gamma}||| e\left\|_{L_{N}^{1}}\right\| x \|_{L_{N}^{\infty}} .
$$

$\left(\right.$ Here $\left.\bar{\Gamma}=\left(\bar{\Gamma}_{1}, \Gamma_{2}, \ldots, \bar{\Gamma}_{N}\right).\right)$

Proof. It follows from Lemma 4 of [1] that each solution $x_{i}(t)$ of the $i$-th component boundary value problem of (5.1), namely,

$$
\begin{gathered}
x_{i}^{\prime \prime}(t)+p_{i}(t) x_{i}(t)=e_{i}(t), \quad 0<t<\pi \\
x_{i}^{\prime}(0)=x_{i}^{\prime}(\pi)=0,
\end{gathered}
$$

satisfies the inequality

$$
\left(1-\frac{\pi^{2}}{4} \bar{\Gamma}_{i}\right)\left\|x_{i}^{\prime \prime}\right\|_{L_{N}^{1}}^{2} \leq 2\left\|e_{i}\right\|_{L^{1}}\left\|x_{i}^{\prime \prime}\right\|_{L^{1}}+\bar{\Gamma}_{i}\left\|e_{i}\right\|_{L^{1}}\left\|x_{i}\right\|_{L^{\infty}}
$$

for each $i=1,2, \ldots, N$. Noting that $\max _{1 \leq i \leq n} \bar{\Gamma}_{i} \leq|\bar{\Gamma}|$, we get

$$
\left(1-\frac{\pi}{4}|\bar{\Gamma}|\right)|| x_{i}^{\prime \prime}\left\|_{L^{1}}^{2} \leq 2\right\| e_{i}\left\|_{L^{1}}\right\| x_{i}^{\prime \prime}\left\|_{L^{1}}+|\bar{\Gamma}|\right\| e_{i}\left\|_{L^{1}}\right\| x_{i} \|_{L^{\infty}}
$$

for each $i=1,2, \ldots, N$. On adding all these inequalities and using the Cauchy-Schwarz inequality in $\mathbf{R}^{N}$ we get that

$$
\left(1-\frac{\pi^{2}}{4}|\bar{\Gamma}|\right)\left\|x^{\prime \prime}\right\|_{L_{N}^{1}}^{2} \leq 2\|e\|_{L_{N}^{1}}\left\|x^{\prime \prime}\right\|_{L_{N}^{1}}+|\bar{\Gamma}|\|e\|_{L_{N}^{1}}\|x\|_{L_{N}^{\infty}} .
$$

Hence the lemma follows.

Theorem 3 Let $\Gamma=\left(\Gamma_{1}, \Gamma_{2}, \ldots, \Gamma_{N}\right) \in L^{1}\left([0, \pi], \mathbf{R}^{N}\right)$ be such that $|\bar{\Gamma}|<\frac{4}{\pi^{2}}$. Let $f=\left(f_{1}, f_{2}, \ldots, f_{N}\right)$ : $[0, \pi] \times \mathbf{R}^{N} \rightarrow \mathbf{R}^{N}$ be as in Theorem 2. Then for every $e \in L^{1}\left([0, \pi], \mathbf{R}^{N}\right)$ with $a_{i} \leq \bar{e}_{i} \leq A_{i}$ for each $i=1,2, \ldots, N$, the boundary value problem

$$
\begin{gathered}
x^{\prime \prime}(t)+f(t, x(t))=e(t), 0<t<\pi, \\
x^{\prime}(0)=x^{\prime}(\pi)=0,
\end{gathered}
$$

has at least one solution.

Proof. Define $F=\left(F_{1}, F_{2}, \ldots, F_{N}\right):[0, \pi] \times \mathbf{R}^{N} \rightarrow \mathbf{R}^{N}, E:[0, \pi] \rightarrow \mathbf{R}^{N}$ and $E=\left(E_{1}, E_{2}, \ldots, E_{N}\right)$ as in the proof of Theorem 2. Then the boundary value problem (5.4) is equivalent to the boundary value problem

$$
x^{\prime \prime}(t)+F(t, x(t))=E(t), \quad 0<t<\pi,
$$




$$
x^{\prime}(0)=x^{\prime}(\pi)=0
$$

Also

$$
\lim \sup _{\left|x_{i}\right| \rightarrow \infty} \frac{F_{i}(t, x)}{x_{i}} \leq \Gamma_{i}(t)
$$

uniformly a.e. in $t \in[0, \pi]$ and

$$
F_{i}(t, x) x_{i} \geq 0,
$$

for a.e. $t \in[0, \pi]$ and all $x \in \mathrm{R}^{N}$ with $\left|x_{i}\right| \geq \max \left(R_{i},-r_{i}\right)$ so that $\Gamma_{i}(t) \geq 0$ for a.e. $t \in[0, \pi]$. Let $\eta=\frac{1}{2 N}\left(\frac{4}{\pi^{2}}-|\bar{\Gamma}|\right)$ so that $|\bar{\Gamma}|+N \eta<\frac{4}{\pi^{2}}$. Proceeding as in the proof of Theorem 2, we can write the boundary value problem (5.5) in the equivalent form

$$
\begin{gathered}
x^{\prime \prime}(t)+g(t, x(t))+h(t, x(t))=E(t), \\
x^{\prime}(0)=x^{\prime}(\pi)=0 .
\end{gathered}
$$

The same degree arguments will imply the existence of a solution for (5.6) if the set of all possible solutions of the family of equations

$$
\begin{gathered}
x^{\prime \prime}(t)+(1-\lambda) \Gamma^{* T}(t) I x(t)+\lambda g(t, x(t))+\lambda h(t, x(t))=\lambda E(t) \\
x^{\prime}(0)=x^{\prime}(\pi)=0,
\end{gathered}
$$

$\lambda \in(0,1)$, is a priori bounded in $X=C\left([0, \pi], \mathbf{R}^{N}\right)$ independently of $\lambda$. Here $\Gamma^{*}(t)=\left(\Gamma_{1}^{*}(t), \ldots, \Gamma_{N}^{*}(t)\right)$ with $\Gamma_{i}^{*}(t)=\Gamma_{i}(t)+\eta, i=1,2, \ldots, N, t \in[0, \pi]$.

We note that $g=\left(g_{1}, g_{2}, \ldots, g_{N}\right)$ in $(5.6)$ is such that $g_{i}(t, x)=\tilde{\gamma}_{i}(t, x) x_{i}$. If we write $\tilde{\gamma}(t, x)=$ $\left(\tilde{\gamma}_{1}(t, x), \ldots, \tilde{\gamma}_{N}(t, x)\right)$, then

$$
g(t, x)=\tilde{\gamma}(t, x)^{T} I x .
$$

We see that

$$
0 \leq(1-\lambda) \Gamma_{i}^{*}(t)+\lambda \tilde{\gamma}_{i}(t, x(t)) \leq \Gamma_{i}^{*}(t)
$$

for $i=1,2, \ldots, N$ in view of (4.40) with

$$
\begin{aligned}
\left|\bar{\Gamma}^{*}\right| & =\left\{\sum_{i=1}^{N}\left(\bar{\Gamma}_{i}+\eta\right)^{2}\right\}^{1 / 2} \leq\left(\sum_{i=1}^{N} \bar{\Gamma}_{i}^{2}\right)^{1 / 2}+\sqrt{N} \eta \\
& \leq|\bar{\Gamma}|+\eta N<\frac{4}{\pi^{2}} .
\end{aligned}
$$

Also, since

$$
\|E(t)-h(t, x(t))\|_{L_{N}^{1}} \leq\|E\|_{L_{N}^{1}}+\sum_{i=1}^{N}\left\|m_{i}\right\|_{L^{1}},
$$

it follows from Lemma 5 that

$$
\begin{gathered}
\left(1-\frac{\pi^{2}}{4}\left|\bar{\Gamma}^{*}\right|\right)\left\|x^{\prime \prime}\right\|_{L_{N}^{1}}^{2} \leq 2\left(\|E\|_{L_{N}^{1}}+\sum_{i=1}^{N}\left\|m_{i}\right\|_{L^{1}}\right)\left\|x^{\prime \prime}\right\|_{L_{N}^{1}} \\
+\left|\bar{\Gamma}^{*}\right|\left(\|E\|_{L_{N}^{1}}+\sum_{i=1}^{N}\left\|m_{i}\right\|_{L^{1}}\right)\|x\|_{L_{N}^{\infty}}
\end{gathered}
$$


As in the proof of Theorem 2, we have that there exist constants $C_{1} \geq 0$ and $C_{2} \geq 0$, independent of $\lambda \in(0,1)$ such that

$$
\begin{aligned}
|\bar{x}| \leq\|x\|_{L_{N}^{\infty}} & \leq C_{1}+C_{2}\|\tilde{x}\|_{H_{N}^{1}} \\
& =C_{1}+C_{2}\left\|x^{\prime}\right\|_{L_{N}^{2}} \\
& \leq C_{1}+C_{2} \frac{\pi}{2}\left\|x^{\prime \prime}\right\|_{L_{N}^{1}} .
\end{aligned}
$$

It then follows from (5.8) and (5.9) that there exists a constant $C_{3} \geq 0$, independent of $\lambda \in(0,1)$, such that

$$
\left\|x^{\prime \prime}\right\|_{L_{N}^{1}} \leq C_{3}
$$

and, hence, from (5.9) again,

$$
\|x\|_{L_{N}^{\infty}} \leq C_{1}+C_{2} \frac{\pi}{2} C_{3}
$$

Hence the theorem holds.

Remark 2. If there is no $L^{\infty}$-resonance (i.e., $\Gamma_{\alpha}=\Gamma_{\infty}=0$ ), Theorem 3 improves the condition on $\bar{\Gamma}$ to $|\bar{\Gamma}| \leq \frac{4}{\pi^{2}}$ compared to Theorem 2 , where $|\bar{\Gamma}|<\frac{3}{\pi^{2}}$.

Remark 3. If $p(t)=\left(p_{1}(t), \ldots, p_{N}(t)\right) \in L^{1}\left([0, \pi], \mathbf{R}^{N}\right)$ in Lemma 5 satisfies, additionally, for a given $\eta>0, p_{i}(t) \geq \eta>0$ for a.e. $t \in[0, \pi], i=1,2, \ldots, N$ and $|\bar{\Gamma}|<\frac{4}{\pi^{2}}$, it follows easily from the inequality (5.3) that the boundary value problem (5.1) has at most one solution.

\section{References}

1. GUPTA, C. P., Two-Point Boundary Value Problems Involving Reflection of the Argument, Internat. J. Math. and Math. Sci. 10 (1987) 361-371.

2. GUPTA, C. P. and MAWHIN, J., Asymptotic Conditions at the First Two Eigenvalues for the Periodic Solutions of Liénard Differential Equations and an Inequality of E. Schmidt, Zeit. für Analysis und ihre Anwendungen 3 (1984) 33-42.

3. IANACCI, R. and NKASHAMA, M. N., Periodic Solutions for Some Forced Second Order Liénard and Duffing Systems, Bolletino U.M.I 4-B (1985) 557-568.

4. KUFNER, A. and KADLEC, J., Fourier Series, Academia, Prague, 1971.

5. MAWHIN, J., Topological Degree Methods in Nonlinear Boundary Value Problems, CBMSRegional Conference Series in Math., No. 40, American Mathematical Society, Providence, R.I., 1979.

6. MAWHIN, J., Landesman Lazer Type Problems for Nonlinear Equations, Conf. Sem. Univ. Bari, No. 147 (1977).

7. MAWHIN, J., Compacité, Monotonie et Convexité dans l'étude de Problèmes aux Limites Semi-Lineaires, Semi. Anal. Moderne 19 (1981) Université de Sherbrooke, Quebec, Canada.

8. ROUCHE, N. and MAWHIN, J., Ordinary Differential Equations, Stability and Periodic Solutions, Pitman, Boston, 1980. 


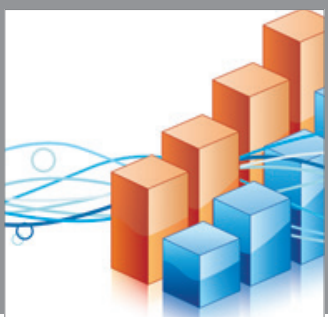

Advances in

Operations Research

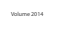

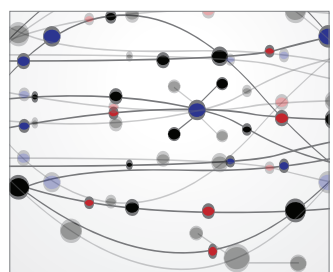

\section{The Scientific} World Journal
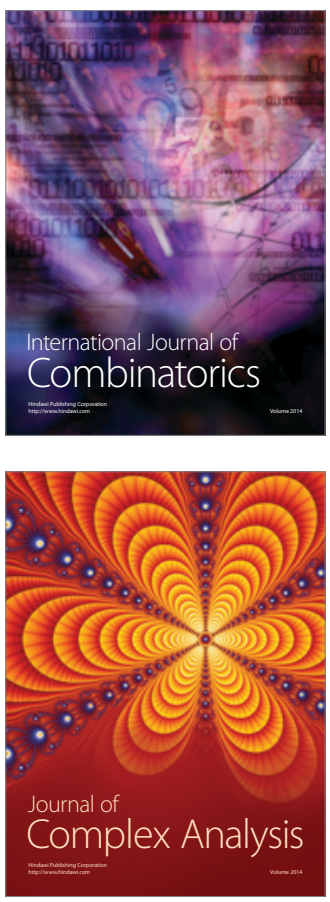

International Journal of

Mathematics and

Mathematical

Sciences
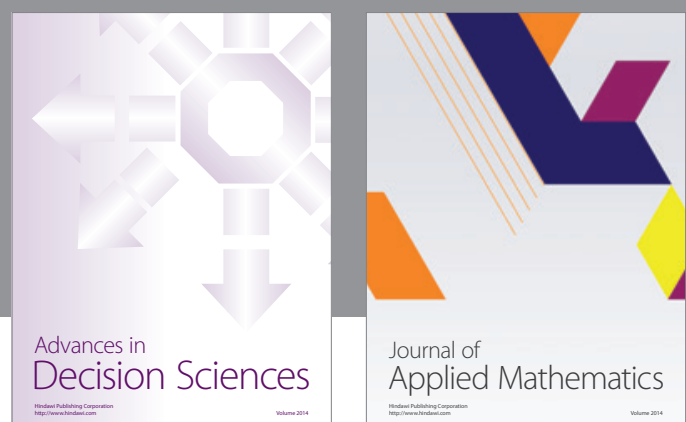

Journal of

Applied Mathematics
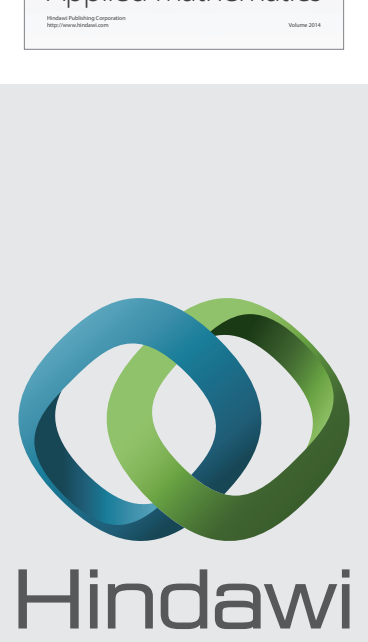

Submit your manuscripts at http://www.hindawi.com
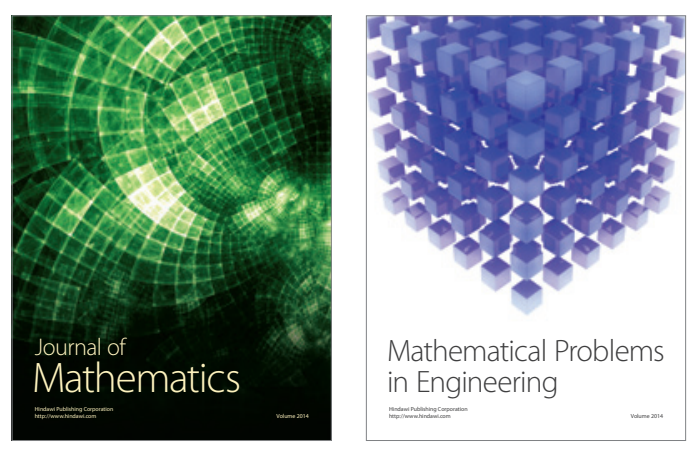

Mathematical Problems in Engineering
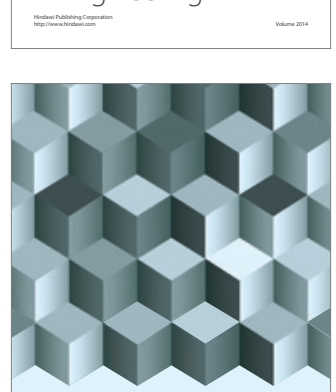

Journal of

Function Spaces
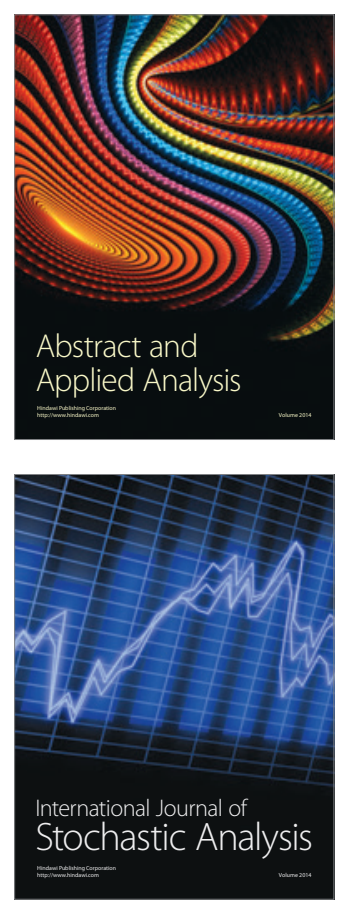

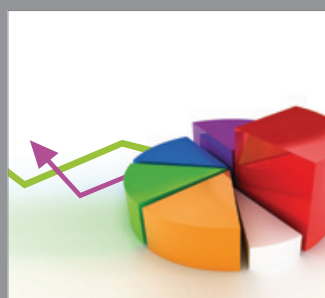

ournal of

Probability and Statistics

Promensencen
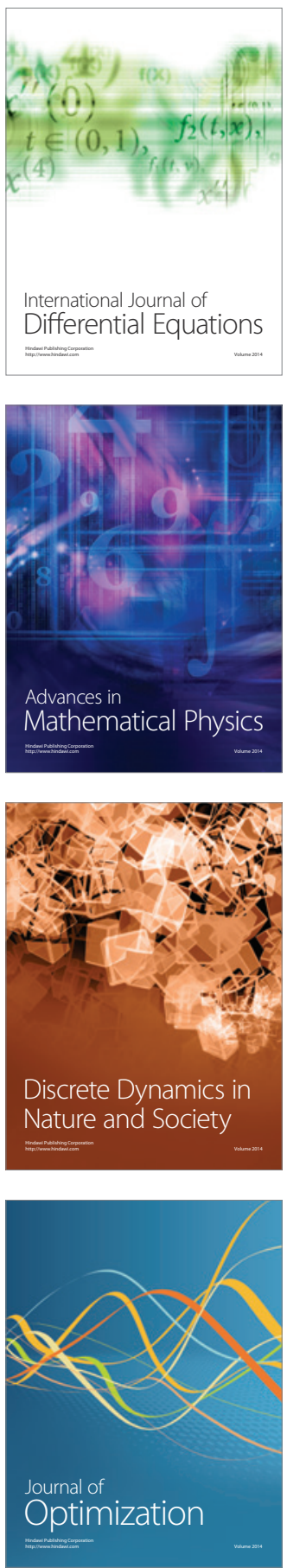